\title{
A Student Physical Therapist's Perspective on her Efficacy Providing Culturally Competent Care: A Reflective Case Study
}

Anna S. Kelly

Mercer University, annakelly1100@gmail.com

Deborah M. Wendland

Mercer University, wendland_dm@mercer.edu

Follow this and additional works at: https://nsuworks.nova.edu/ijahsp

Part of the Curriculum and Instruction Commons, and the Physical Therapy Commons

\section{Recommended Citation}

Kelly AS, Wendland DM. A Student Physical Therapist's Perspective on her Efficacy Providing Culturally Competent Care: A Reflective Case Study. The Internet Journal of Allied Health Sciences and Practice. 2021 Oct 01;19(4), Article 10.

This Case Study is brought to you for free and open access by the College of Health Care Sciences at NSUWorks. It has been accepted for inclusion in Internet Journal of Allied Health Sciences and Practice by an authorized editor of NSUWorks. For more information, please contact nsuworks@nova.edu. 


\title{
A Student Physical Therapist's Perspective on her Efficacy Providing Culturally Competent Care: A Reflective Case Study
}

\begin{abstract}
Purpose: Cultural differences and personal biases can affect the way a clinician provides care and the way in which patients receive it. The Commission on Accreditation in Physical Therapy Education (CAPTE) designates cultural competence as one of the professional practice expectations for physical therapy students, although no standardized blueprint exists for Doctor of Physical Therapy (DPT) programs. This case report will illustrate how reflection can be used to facilitate a student's growth into becoming more prepared to practice in a culturally aware and effective way. In addition, this report seeks to provide a student's perspective on her efficacy providing culturally competent care, given her training and education through her DPT program. Method: This case report will present the interactions between a student physical therapist (SPT) and a culturally and linguistically diverse patient throughout a physical therapy plan of care. Subsequently, the reflections by the SPT on these interactions will be presented, and themes such as communication barriers, power dynamics, and patient expectations will be explored. Results: Through the student's self-reflection, she determined both her strengths and areas needing improvement in providing an adequate cross-cultural interaction, in addition to delineating how her preparation in school affected these areas. Her strengths included 1) vulnerability to recognize her own shortcomings, and 2) a desire to create a meaningful connection with the patient. The areas needing improvement included 1) providing an open space for the patient to speak, and 2) setting patient expectations. The student's DPT program facilitated her preparedness in cultural competence by 1 ) providing lecture content promoting cultural awareness, 2) encouraging reflection, and 3) hosting an event where the DPT students were able to work with international students of limited-English proficiency. Conversely, barriers to the student's preparedness included 1) no mandatory community service reaching minority populations, and 2) no opportunity for an abroad immersion experience. Conclusions: Two main themes were drawn from the student's reflection. First, continual self-evaluation and vulnerability are imperative to the process of becoming a culturally competent provider. Second, clinical experience dedicated to providing care to minority populations appears to be effective in fostering cultural competency in DPT students.
\end{abstract}

\section{Author Bio(s)}

Anna S. Kelly, SPT is a Doctor of Physical Therapy candidate at Mercer University in Atlanta, GA.

Deborah M. Wendland, PT, DPT, PhD, is an Associate Professor of Physical Therapy in the College of Health Professions at Mercer University in Atlanta, GA. She is also a licensed physical therapist in the state of Georgia and a certified pedorthist.

\section{Acknowledgements}

We would like to thank Dr. Mary Anna Djire for providing guidance as a clinical instructor throughout the reflection process and for providing an open space to discuss topics such as cultural awareness. 


\title{
1IVAHSP \\ The Internet Joumnal of Allied Health Sciences and Practice \\ Dedicated to allied health professional practice and education \\ Vol. 19 No. 4 ISSN 1540-580X
}

\section{A Student Physical Therapist's Perspective on her Efficacy Providing Culturally Competent Care: A Reflective Case Study}

\author{
Anna S. Kelly \\ Deborah M. Wendland \\ Mercer University \\ United States
}

\begin{abstract}
Purpose: Cultural differences and personal biases can affect the way a clinician provides care and the way in which patients receive it. The Commission on Accreditation in Physical Therapy Education (CAPTE) designates cultural competence as one of the professional practice expectations for physical therapy students, although no standardized blueprint exists for Doctor of Physical Therapy (DPT) programs. This case report will illustrate how reflection can be used to facilitate a student's growth into becoming more prepared to practice in a culturally aware and effective way. In addition, this report seeks to provide a student's perspective on her efficacy providing culturally competent care, given her training and education through her DPT program. Method: This case report will present the interactions between a student physical therapist (SPT) and a culturally and linguistically diverse patient throughout a physical therapy plan of care. Subsequently, the reflections by the SPT on these interactions will be presented, and themes such as communication barriers, power dynamics, and patient expectations will be explored. Results: Through the student's self-reflection, she determined both her strengths and areas needing improvement in providing an adequate cross-cultural interaction, in addition to delineating how her preparation in school affected these areas. Her strengths included 1) vulnerability to recognize her own shortcomings, and 2) a desire to create a meaningful connection with the patient. The areas needing improvement included 1) providing an open space for the patient to speak, and 2) setting patient expectations. The student's DPT program facilitated her preparedness in cultural competence by 1) providing lecture content promoting cultural awareness, 2) encouraging reflection, and 3) hosting an event where the DPT students were able to work with international students of limited-English proficiency. Conversely, barriers to the student's preparedness included 1) no mandatory community service reaching minority populations, and 2) no opportunity for an abroad immersion experience. Conclusions: Two main themes were drawn from the student's reflection. First, continual self-evaluation and vulnerability are imperative to the process of becoming a culturally competent provider. Second, clinical experience dedicated to providing care to minority populations appears to be effective in fostering cultural competency in DPT students.
\end{abstract}

Keywords: cultural competence, physical therapy, Doctor of Physical Therapy 


\section{INTRODUCTION}

The successful delivery of physical therapy services extends beyond knowledge and skill. Communication and sound interpersonal skills play a vital role in providing effective patient-centered care; however, cultural differences and personal biases can affect the way a clinician provides care and also the way in which a patient receives it. Cross defines culture as an "integrated pattern of human behavior that includes thoughts, communications, actions, customs, beliefs, and values" held by a "racial, ethnic, religious, or social group." Because culture can influence patient and provider expectations of each other, a mismatch in these expectations can be problematic for patient outcomes and the patient experience. ${ }^{2}$ With this assumption, a necessary trait for any healthcare provider is cultural competence or, at a minimum, cultural awareness. The Centers for Disease Control and Prevention (CDC) describe cultural competence in terms of attitudes and behaviors that align with health policy to facilitate effective work across cultures and in culturally diverse situations. ${ }^{3}$

The United States (US) includes a vast and growing minority population. In 2018, the foreign-born population, comprised of anyone who was not a US citizen at birth, totaled 44.8 million, or $13.7 \%$ of the US population. ${ }^{4}$ Considering the number of American citizens born to foreign-born individuals, the cultural diversity of the US population becomes even more clear. Projections from the 2010 US Census predicted that the non-Hispanic white population will no longer make up the majority in the US by 2043 , creating what former Director of the US Census Bureau Thomas Mesenbourg described as a "plurality nation." ${ }^{5}$ In regard to language use, half of the nation's foreign-born residents were reported to have limited English-speaking ability. ${ }^{6}$ Language, inseparable from culture, may be the first identifier to healthcare providers that they are providing care to a patient with differing customs and beliefs.

The past decade in the United States has been marked by increasing efforts toward achieving equality and encouraging social responsibility. In healthcare, the first Healthy People objectives were published in 1980 to be achieved by 1990 with goals to improve the health of people nationwide. Beginning with Healthy People 2000 and continuing with each decade iteration, the reduction or elimination of health disparities and promotion of health equity has been central. ${ }^{6}$ The Healthy People initiative has carved the way for many health agencies to establish their stance on inclusivity and develop strategies to promote cultural competency in their professions. Among that group, the American Physical Therapy Association (APTA) expresses its role in health equity with its mission statement of "building a community that advances the profession of physical therapy to improve the health of society." "The word "society" is inclusive. The APTA offers three guiding principles that support the profession's commitment to cultural competence. These principles emphasize "consumer-centricity," access and equity, and advocacy. ${ }^{7}$

Doctor of Physical Therapy (DPT) programs nationwide are challenged with the task of developing curricula that align with the professional mission. While the desire to cultivate a culturally competent profession is clear, guidance provided by the APTA on how to achieve this goal is non-prescriptive. Because the mechanism of execution is not prescribed, DPT programs are widely variable in the strategies they employ to prepare students for cultural competence. Didactic course content regarding cultural competence training are commonplace throughout DPT curricula, as the Commission on Accreditation in Physical Therapy Education (CAPTE) designates standards that reflect the need for cultural competence as a professional practice expectation. ${ }^{8}$ Beyond the didactic elements, alternative avenues to cultivate cultural competence in student cohorts exist, such as immersion experiences, service-learning, and community service. Several researchers in physical therapy education have explored the benefits of a short-term immersion experience, where DPT students spend several days or weeks providing physical therapy services in countries that are culturally divergent from the United States. ${ }^{9,10,11}$ One positive outcome of immersion described in the literature is the effect of combatting ethnocentrism, meaning judging other cultures through comparison to one's own culture. 9,10,12 More common and feasible than immersion programs are community-based projects in which DPT programs offer both volunteer opportunities and/or mandatory participation in pro-bono work with groups that include culturally diverse populations. ${ }^{13}$ Another documented method of promoting cultural competence is refugee screening programs, where students conduct physical therapy screenings on culturally and linguistically diverse individuals. ${ }^{13,14}$

To further assess the promotion of cultural competence, some investigators have studied the perceptions of DPT students (SPT) on their preparedness to be culturally competent providers. This is a critical consideration given the range of approaches to cultural competency education in entry-level programs. One study reported that entry-level physical therapy students felt unprepared to navigate cross-cultural interactions in clinic, citing a lack of clinical preparation, understanding behind cultural themes, and accessible resources as the pitfalls to their preparation..$^{15}$ Conversely, Hillard et al. showed that many students, after having completed the clinical education portion of their program's curriculum, demonstrated improved emotional resilience, openness to new beliefs and attitudes, and effectiveness with verbal and nonverbal communication. ${ }^{16}$ However, the article mentioned that not all students were placed in clinical sites that consistently worked with minority populations, meaning these gains towards cultural competency were not shared equally among the cohort. Furthermore, these studies did not detail the mechanism by which SPTs learn cultural competence during regular clinical affiliations. 
As DPT students are subject to variability of cultural competency preparation, the purpose of this case report is to illustrate how reflection can be used to facilitate a student's growth into becoming more prepared to practice in a culturally aware and effective way. Additionally, this report seeks to provide a student's perspective on her efficacy providing culturally competent care, given her training and education through her DPT program. A brief outline of the didactic coursework related to cultural competence provided by the student's DPT program is provided in Table 1.

Table 1. Didactic Content

\begin{tabular}{|l|l|}
\hline \multicolumn{2}{|c|}{ Didactic Content on Cultural Competence Provided by the Student's DPT Program } \\
\hline $\begin{array}{l}\text { PT 502: Leadership } \\
\text { Development I: Foundations }\end{array}$ & $\begin{array}{l}\text { Lecture: "Strategies for Improving Healthcare Communication across Language and Culture" } \\
\text { Lecture: "Communication Across the Generations" } \\
\text { Activity: History taking practice with English as a second language (ESL) students with written } \\
\text { reflection on the experience }\end{array}$ \\
\hline $\begin{array}{l}\text { PT 632: Leadership } \\
\text { Development II: Health } \\
\text { Policy }\end{array}$ & $\begin{array}{l}\text { Lecture: "Social Determinants of Health and Health Literacy" } \\
\text { Required Reading: "Quick Guide to Health Literacy" by the U.S. Department of Health and } \\
\text { Human Services }\end{array}$ \\
\hline Other & $\begin{array}{l}\text { Formal reflection processes after practical examinations, community engagement events, and } \\
\text { clinical experiences throughout the curriculum }\end{array}$ \\
\hline
\end{tabular}

Note: Coursework outlined above limited to topics that specifically discuss or incorporate cultural competence.

\section{METHOD}

Mercer University's International Review Board approved the production of this case report, which discusses the interactions between an SPT and a culturally and linguistically diverse patient throughout a physical therapy plan of care. The SPT's reflection on the experience and her efficacy of providing an inclusive environment will also be discussed. The patient's relevant history taken during the initial evaluation is listed in Table 2.

Table 2. Patient History

\begin{tabular}{|l|l|}
\hline \multicolumn{2}{|c|}{ Relevant Patient History } \\
\hline Age/Gender & 66 -year-old female \\
\hline Ethnicity & Black, Haitian descent \\
\hline Primary language & French-Creole, limited English-proficiency \\
\hline $\begin{array}{l}\text { Reason for Seeking Physical } \\
\text { Therapy }\end{array}$ & $\begin{array}{l}\text { Low back pain and bilateral knee pain that prevented her from tolerating a full day of } \\
\text { standing at work }\end{array}$ \\
\hline
\end{tabular}

\section{Data Collection}

Data collection for this case report took place over eight weeks, while the patient received physical therapy services from the SPT under the guidance of a clinical instructor $(\mathrm{Cl})$. Data was collected qualitatively through the SPT's self-guided reflection, in addition to input provided by the $\mathrm{Cl}$. The majority of data was derived from the SPT's reflection of the initial evaluation and can be divided into two sections: the SPT's impression of the patient and the SPT's impression of her own performance. Additionally, Table 3 provides a summary of the lessons learned by the SPT over the eight weeks of collective interactions with the patient. Each lesson learned was a direct response to deliberate and guided self-reflection by the SPT.

\section{Impression of the Patient}

The impression of the patient was developed both in real-time and after the initial reflection. The most obvious identifier that the patient was culturally diverse from the SPT was the patient's limited-English proficiency (LEP). While the patient seemed to have responded with appropriate answers to all of the SPT's questions, indicating a general understanding of what was being asked, she required increased time for word-finding and kept responses short and simple. 
One of the major insights from the initial reflection on the initial visit was the hypothesis of a potential power distance between the SPT and the patient. Power distance, a term coined by Geert Hofstede, is a particular cultural trait that describes the acceptance of unequal power distribution among members of a society. ${ }^{17}$ An example of a power distance in healthcare is when members of certain cultures view medical professionals as authoritative figures, and all directions by medical professionals should be followed without question. Two instances reinforced the hypothesis that the patient held a hierarchical view of the SPT as a healthcare provider. First, in the objective portion of the exam, the patient seemed hesitant to report pain symptoms. For example, the patient would wince during certain tests and measures but would not verbalize her pain unless she was explicitly asked if the examination measure was painful. The SPT thought that perhaps 1) the patient did not want to reveal that the "doctor" was hurting her, or 2) she should only speak when spoken to when in a medical office. Further evidence supporting the power distance hypothesis came from one of the patient's verbal statements in response to the SPT's attempt to determine a directional preference for the patient's back pain. The SPT asked the patient to lie in prone to assess her tolerance to lumbar extension. When the SPT asked "Is this okay?", the patient responded with "I have to try." This response demonstrated a misunderstanding about the intention of the SPT's questions. The patient appeared to perceive this interaction as the SPT prescribing the exercise, rather than an attempt to understand behaviors that aggravated or alleviated her symptoms. Both the hesitancy to report pain and the verbal statement led the SPT to believe that the patient did not feel that her input would be welcomed in the prescription of physical therapy interventions. Instead, perhaps, the patient expected to show up and be told what to do.

\section{Impression of Own Performance}

Through reflection, the SPT realized that there were several missed opportunities during the cross-cultural interaction. For example, one of the missed opportunities stemmed from the SPT's insecurities about communicating with someone in the presence of a language barrier. The patient required increased time for word-finding, which often led to long pauses. Uncomfortable with the pace of the conversation, the SPT would try to fill the gaps by guessing which words the patient meant so that they could continue with further questioning. Additionally, the slower pace of the interview led the SPT to refrain from asking certain follow-up questions, even if the SPT felt she needed clarification on what the patient had reported. Clearly, this led to an ineffective and incomplete subjective exam that led the SPT to be unsure about differential diagnoses, while also likely making the patient feel unheard. Because communication throughout the interview was such a labored effort, the SPT also failed to build rapport with the patient by asking fewer "get-to-know-you" type questions than she typically would with patients with good English proficiency. Failing to make a personal connection on the first day may have reinforced the potential power dynamic between the SPT and the patient.

Another missed opportunity was failing to take the time to intentionally set expectations for physical therapy. The SPT felt that at the beginning of the session, she should have gone over the objectives for the visit (i.e. obtain a history, test hypotheses through objective tests and measures, provide initial interventions). More importantly, the SPT should have thoroughly explained that the intent of the objective portion of the exam was to reproduce the patient's pain so that a source of symptoms could be determined. Failing to do so may have directly contributed to the patient's hesitancy to report her pain. Physical therapy is a collaborative process, and the SPT felt that she had failed to promote such collaboration by missing the opportunity to set expectations for the visit.

\section{Response to Reflection}

Through this reflection, the SPT and her $\mathrm{Cl}$ were able to devise a plan to implement strategies to improve communication and collaboration with the patient for the remainder of her plan of care. The first goal was to build rapport with the patient as soon as possible. The SPT planned to ask questions about the patient's hobbies and family, as she would with any other patient she treated. Doing so may help to dissipate the potential power dynamic between the two. Next, the SPT wanted to be sure that she would allow sufficient time for the patient to form her thoughts and produce a complete response to questions asked during the session. This required the SPT to be welcoming of a slower pace of conversation. To further enhance the quality of communication, the SPT sought to explain the purpose behind each examination measure or intervention. The SPT hoped that relating elements of the session to the patient's pain and functional complaints would relieve the patient's anxiety about physical therapy and improve her participation. In addition to the initial reflection after the evaluation, the SPT learned over multiple follow-up visits that the use of slang was prohibitive of effective communication. The SPT had to be conscious of her use of slang throughout their interactions and also recognize when the SPT's words were not received so that she could explain using different words or phrases. For example, the SPT would use the the word "homework" to reference the patient's home exercise program, but eventually the SPT realized that this word did not translate adequately, leading to the patient failing to realize that she was being asked to do exercises at home. Through these efforts, each follow up session became more productive than the last, and more importantly, the patient began to see functional improvements. 


\section{RESULTS}

This case report was designed to serve as an illustration of a student's learning process throughout a cross-cultural interaction, including its miscues, so that other students and clinicians may feel empowered to have the same vulnerability through reflection on their own performances. In addition, the case report adds to the literature the perspective of a novice clinician in her preparedness to be culturally competent, a professional standard set by the APTA and CAPTE, as it relates to her DPT education.

\section{Strengths}

While there were several missed opportunities, the student demonstrated two major strengths that ultimately led to a successful plan of care. The SPT had 1) the vulnerability required to reflect and 2) a desire to make a meaningful connection with the patient.

This case report would not have come to fruition had the student not taken the time to reflect on her performance after delivering the initial evaluation to the patient. The SPT recognized that the session did not go smoothly and dedicated time to exploring the potential barriers that were present to providing the patient with the most inclusive care. More importantly, the SPT took responsibility for creating those barriers, rather than trying to shift blame onto the patient. It was her vulnerability in admitting her insecurities and shortcomings that led to her growth as a clinician who seeks to provide culturally competent care. Tervalon \& Murray-Garcia stress the importance of seeking "cultural humility" rather than cultural competence. ${ }^{18}$ They argue that mastering cultural competence may never be an achievable goal; however, having the humility to recognize one's own biases will be the greatest catalyst towards providing more inclusive care. Thus, the benefit of a lifelong commitment to humility is the formation of reflective clinicians who provide patient-focused care. ${ }^{18}$ Reflection is encouraged throughout physical therapy school, but it is also necessary to add critical self-evaluation to one's clinical reasoning toolbox to ensure continued growth in all aspects of care. It is also important to be encouraged and supported in this process by the clinical arm of the clinical education team, as was the student's $\mathrm{Cl}$.

More important than simply recognizing her shortcomings, the SPT had the strong desire to create positive change so that future interactions felt more inclusive. Campinha-Bacote describes this as "cultural desire," or the motivation of a provider to want to engage in the process of becoming culturally competent. ${ }^{19}$ Dissatisfied with her performance, the SPT looked inward to develop strategies to earn the patient's trust. The SPT wanted physical therapy to be a positive experience, in which the patient could heal in a safe environment. In a study that examined patient perspectives on the cultural competency of their providers, patients reported that having a meaningful connection with their provider was essential to their commitment to rehabilitation. ${ }^{20}$ Ultimately, kindness and respect can be the greatest tools for ensuring an effective patient-care experience.

\section{Areas needing improvement}

One clear theme from the SPT's impression of her performance was the effect of the communication barrier on the patient-therapist interactions. Several of the student's own realizations on how to provide more sensitive care are consistent with a report by Li et al. that delineates strategies healthcare providers can utilize to address barriers in communication. ${ }^{21}$ The SPT realized that she prevented open communication because of her propensity to "fill in the gaps" of conversation by guessing the words for which the patient was searching. As the SPT and the patient became more familiar, it became apparent that the patient actually had decent English-speaking skills. Communication could be effective if the SPT provided adequate time for the patient to articulate her thoughts and responses. For cases such as this, Li et al. suggest a slower pace of speaking and use of shorter sentences so that patients have the space to process the clinician's words. ${ }^{21}$ Doing so may allow the patient to feel more confident in responding. In addition, it is important to recognize the many nuances of the English language, and even proficient English speakers may be confused by common terms, including slang, used in the US; therefore, clinicians should always try to provide context to their words. ${ }^{21}$

Additionally, the SPT's failure to set patient expectations at the beginning of the session likely caused confusion throughout the physical therapy process. Grandpierre et al. illuminated that patients desire an understanding behind rehabilitation services, such as the purpose of therapy, how long it will take, and the benefits of compliance. ${ }^{20}$ Providing these explanations demonstrates respect for any patient but is particularly necessary when treating the culturally and linguistically diverse patient to reduce barriers for an effective interaction.

\section{Facilitators of Preparedness}

Pivotal to the SPT's recognition of cultural barriers was the SPT's education related to cultural competence provided by her DPT program. In a first-year course dedicated to leadership and professional development, the SPT received a series of lectures related to cultural competence outlined in Table 1. These lectures offered guidance to navigate both generational differences and cultural differences. Additionally, these lectures provided strategies for implementing better communication, especially with those who are 
linguistically diverse. Included within these lectures, information about Geert Hofstede's cultural dimensions and other cultural characteristics that can change the way an individual receives healthcare widened the student's perspective of the impact of culture on physical therapy care. These lessons improved the SPT's sensitivity to a potential power dynamic issue with her patient.

In addition to in-class lectures, the same course provided an opportunity for the students to practice communication with individuals with LEP. The course coordinator had developed an event with the university's English Language Institute, which offers ESL to international students. The event was designed to simulate a health screening, where the DPT students were able to practice taking a subjective history of a person with LEP, and the ESL students were able to practice holding medical conversations in English. After the exercise, the students were encouraged to reflect on their 1) use of formal and informal conversation, 2) use of colloquial and idiomatic language, 3) pace of communication, and 4) ability to create space for the client to find a way to speak. Taken from the SPT's written reflection from the event, the SPT reported that "having the [lecture regarding cultural competence] prior to the experience was helpful, but actually having the face-to-face interaction was invaluable." This excerpt reveals how inperson experience is crucial to developing cultural competence in physical therapy students.

\section{Barriers to Preparedness}

Participation in community service events for minority populations was not required in the curriculum of the student's DPT program; however, community service opportunities did exist for students who were interested in volunteering. The DPT program was closely associated with a health clinic for medically underserved people. The majority of the individuals receiving healthcare were members of minority groups, many of whom did not speak English as their primary language. Regretfully, the SPT of this case report never participated in this clinic and missed out on an opportunity to practice her communication skills with culturally and linguistically diverse individuals. While this missed opportunity falls on the student, a requirement to participate in this clinic, if only for one day, would have fortified the DPT program's attempt to foster cultural competence. Panzarella et al. analyzed one accredited program's curriculum for its contribution to fostering cultural competence in its students and delineated the methods that were mandatory or voluntary. ${ }^{14}$ The study discovered that the students' voluntary participation in a pro bono service clinic served as a major contributor to the student's cultural competence development, as the clinic offered the student an opportunity to consistently interact across cultures. ${ }^{14}$ Knowing this, DPT programs who are committed to developing a culturally-sound curriculum would benefit from transitioning these types of experiences from being voluntary to being mandatory. Further, Panzarella et al. affirm that when curricula include experiential learning that exposes the students to different cultures, the result vastly improved students' perception of their own cultural competence. ${ }^{14}$

In addition, immersion abroad experiences, now common within DPT programs, were not offered to the student. Researchers and participants of immersion experiences argue that immersion into a culture may be the most efficient way to foster cultural competence in students. ${ }^{9,10,11}$ Even short-term immersion experiences, lasting 1-2 weeks, can provide substantial benefits, such as minimizing the students' own ethnocentric views. ${ }^{9,10}$ Inherent to ethnocentrism is the belief that one's culture is superior to those of other groups. ${ }^{12}$ Unchecked, this belief poses obvious barriers to providing culturally competent healthcare services. Illustrating the effect of an immersion experience on an ethnocentric view, Grzelak et al. reported that a DPT student at the end of an 8-day immersion experience "felt better able to embrace the culture for its own sake rather than in comparison to her own." 10 Moreover, immersion experiences can ignite the desire for students to participate in global initiatives and inspire students to continue to learn about new cultures; therefore, offering international opportunities to students may promote the desire to seek similar opportunities as practicing clinicians. ${ }^{10}$ Other cited benefits include development of a professional identity and reinforcement of the importance of advocacy in students. ${ }^{11}$ Dupre \& Goodgold argue that providers cannot truly progress along the cultural competence continuum unless they themselves are in a position to provide healthcare for the culturally diverse. ${ }^{9}$ Understandably, immersion experiences may not be feasible for all DPT programs to offer, considering cost of travel, safety and liability concerns, and lack of established partnerships with international programs; however, the research supports benefits to immersion that are unparalleled by less involved approaches. 9,10

\section{CONCLUSION \\ Implications for PT Practice}

The lessons presented in Table 3 can be used as a quick reference for clinicians seeking guidance on cross-cultural interactions. Moreover, two major take-home messages have been determined through this case study's exploration. First, continual reflection and vulnerability are imperative to the process of becoming a culturally humble and competent provider. This case report echoes the APTA's suggestion for members to "take an honest look at themselves, their own biases, and how those biases may affect the care they provide patients." ${ }^{.7}$ Second, experiential learning that involves the provision of physical therapy services to minority populations appears to be extremely effective in fostering cultural competence in DPT students. While lecture content related to cultural competence education is both productive and valuable, there is no training that can occur in a classroom that sufficiently 
prepares a student in culturally competent care. Rather, the process is a lived, lifelong experience that can only be developed through an accumulation of thousands of cross-cultural interactions.

Table 3. Lessons were taken from initial and ongoing reflection related to patient care.

\section{Lessons Learned}

1. Building rapport, despite language barriers, is important for reinforcing a collaborative relationship with the patient.

2. Be patient and provide a space for the patient to speak.

3. Setting patient expectations for the session should occur at the beginning of the interaction.

4. Always provide context to your words.

5. Avoid using complicated language for the LEP speaker to understand, including idioms and slang (e.g. "scooch" or "homework").

\section{Areas for Further Research}

A paucity of new research exists regarding the implementation of cultural competency strategies in DPT curricula and how such strategies affect student preparedness in being culturally competent providers. As 2021 begins, an opportunity exists for researchers to explore whether the physical therapy profession, as it currently operates, aligns with the new set of objectives for Health People 2030.

\section{REFERENCES}

1. Cross, TL. Towards a culturally competent system of care: a monograph on effective services for minority children who are severely emotionally disturbed. Published by Georgetown University Child Development Center; 1989.

2. Goode TD, Dunne C. Policy Brief 1: Rationale for Cultural Competence in Primary Care. National Center for Cultural Competence. https://nccc.georgetown.edu/documents/Policy_Brief_1_2003.pdf. Published 2003. Accessed Oct 18, 2020.

3. Centers for Disease Control and Prevention. Cultural Competence in Health and Human Services. https://npin.cdc.gov/pages/cultural-competence\#3. Revised Oct 17, 2020. Accessed Oct 18, 2020.

4. Abby Budiman. Key findings about U.S. immigrants. Pew Research Center. https://www.pewresearch.org/facttank/2020/08/20/key-findings-about-u-s-immigrants/. Published Aug 20, 2020. Accessed Feb 11, 2021.

5. United States Census Bureau. U.S. Census Bureau Projections Show a Slower Growing, More Diverse Nation a Half Century from Now. United States Census Bureau.

https://www.census.gov/newsroom/releases/archives/population/cb12-243.html. December 12, 2012. Accessed October 11, 2020.

6. Gambino CP, Acosta YD, Grieco EM. English-speaking ability of the foreign-born population in the United States: 2012. American Community Survey Reports. https://www2.census.gov/library/publications/2014/acs/acs-26.pdf. Published June 2014. Accessed Oct 18, 2020.

7. United States Census Bureau. U.S. Census Bureau Projections Show a Slower Growing, More Diverse Nation a Half Century from Now. United States Census Bureau. https://www.census.gov/newsroom/releases/archives/population/cb12-243.html December 12, 2012. Accessed October 11, 2020.

8. Office of Disease Prevention and Health Promotion. About Healthy People. Office of Disease Prevention and Health Promotion. https://www.healthypeople.gov/2020/About-Healthy-People. Accessed Oct 18, 2020.

9. American Physical Therapy Association. Resources for Teaching Cultural Competence in Physical Therapy Education. American Physical Therapy Association. https://www.apta.org/patient-care/public-health-population-care/culturalcompetence/teaching-cultural-competence. Published Oct 6, 2020. Accessed Oct 18, 2020.

10. Commission on Accreditation for Physical Therapy Education. Evaluative Criteria PT Programs. American Physical Therapy Association. http://www.capteonline.org/uploadedFiles/CAPTEorg/About_CAPTE/Resources/Accreditation_Handbook/EvaluativeCri teria_PT.pdf. Revised Aug 7, 2014. Accessed Nov 23, 2020.

11. Dupre AM, Goodgold S. Development of physical therapy student cultural competency through international community service. J Cult Divers. 2007;14(3):126-134. [PMID 18314814] 
12. Grzelak CR, Glickman L. Reflections on an international immersion experience: a Doctor of Physical Therapy student's perspective. J Phys Ther Educ. 2014;28(1):16-22.

13. Hayward LM, Li L. Promoting and assessing cultural competence, professional identity, and advocacy in physical therapy (DPT) degree students within a community of practice. J Phys Ther Educ. 2015;28(1):23-26.

14. Evans RW. Ethnocentrism is an unacceptable rationale for healthcare policy: a critique of transplant tourism position statements. Am J Transplant. 2008;8(6):1089-1095. [PMID 18444938]

15. Palombaro KM, Dole RL, Black JD. Curricular integration and measurement of cultural competency development in a group of physical therapy students. JOSOTL. 2015;15(5):82-96. DOI: 10.14434/josotl.v15i5.13505

16. Panzarella, KJ. Beginning with the end in mind: evaluating outcomes of cultural competence instruction in a Doctor of Physical Therapy programme. Disabil Rehabil. 2009;31(14):1144-1152. [PMID 19479532]

17. Kraemer TJ. Physical therapist student's perceptions regarding preparation for providing clinical cultural congruent cross-cultural care: a qualitative case study. J Phys Ther Educ. 2001;15(1):36-52. DOl:10.1097/00001416-20010100000008

18. Hillard MJ, Rathsack C, Brannigan P, Sander AP. Exploring the cultural adaptability of doctoral entry-level physical therapist students during clinical education experiences. J Allied Health. 2008;37(3):199-220. [PMID 19753398]

19. Geert Hofstede. The 6-D model of national culture. Geert Hofstede. https://geerthofstede.com/culture-geert-hofstedegert-jan-hofstede/6d-model-of-national-culture/. Accessed October 18, 2020.

20. Tervalon M, Murray-Garcia J. Cultural humility versus cultural competence: a critical distinction in defining physician training outcomes in multicultural education. J Health Care Poor Underserved. 1998;9(2):117-125. [PMID 10073197]

21. Campinha-Bacote $J$. The process of cultural competence in the delivery of healthcare services: a model of care. $J$ Transcult Nurs. 2002;13(3):181-184. [PMID 12113146]

22. Grandpierre V, Milloy V, Sikora L, Fitzpatrick E, Thomas R, Potter B. Barriers and facilitators to cultural competence in rehabilitation services: a scoping review. BMC Health Serv Res. 2018;18(23):1-14. [PMID 29334968]

23. Li C, Abdulkerim N, Jordan CA, Ga Eun Son C. Overcoming communication barrier to healthcare for culturally and linguistically diverse patients. N A J Med Sci. 2017;10(3):103-109. DOI: 10.7156/najms.2017.1003103] 